\title{
On the degree of approximation of continuous functions by a specific transform of partial sums of their Fourier series
}

\author{
Xhevat Z. KrasniqI \\ In memory of Professor László Leindler
}

\begin{abstract}
Using the Mean Rest Bounded Variation Sequences or the Mean Head Bounded Variation Sequences, we have proved four theorems pertaining to the degree of approximation in sup-norm of a continuous function $f$ by general means $\tau_{n, A}^{\lambda}(f)$ of partial sums of its Fourier series. The degree of approximation is expressed via an auxiliary function $H(t) \geq 0$ and via entries of a matrix whose indices form a strictly increasing sequence of positive integers $\lambda:=\{\lambda(n)\}_{n=1}^{\infty}$.
\end{abstract}

\section{Introduction}

Let $\mathbf{S}$ be the class of all real sequences and let $\mathbf{w}:=\left\{w_{n}\right\} \in \mathbf{S}$ be any sequence. The sequence $\mathbf{w}$ is said to be monotone increasing (decreasing) if $w_{n}-w_{n+1} \leq 0\left(w_{n}-w_{n+1} \geq 0\right)$ for all $n \in \mathbb{N} \cup\{0\}$. It is shown in [7] that every zero monotone decreasing sequence is of Rest Bounded Variation ( $R B V S$-class), but not conversely. Moreover, every monotone increasing sequence is of Head Bounded Variation ( $H B V S$-class), but not conversely (see $[8])$.

A sequence $\mathbf{w}:=\left\{w_{n}\right\}$ of non-negative numbers tending to zero is called a sequence of Rest Bounded Variation, or briefly $\mathbf{w} \in R B V S$, if it has the property

$$
\sum_{n=m}^{\infty}\left|w_{n}-w_{n+1}\right| \leq K(\mathbf{w}) w_{m}
$$

for all natural numbers $m$, where $K(\mathbf{w})$ is a constant depending only on w.

Received December 14, 2019.

2020 Mathematics Subject Classification. 42A10, 41A25, 40 G05.

Key words and phrases. Matrix transformation, degree of approximation, Fourier series, modulus of continuity.

https://doi.org/10.12697/ACUTM.2021.25.01 
A sequence $\mathbf{w}:=\left\{w_{n}\right\}$ of non-negative numbers will be called a sequence of Head Bounded Variation, or briefly $\mathbf{w} \in H B V S$, if it has the property

$$
\sum_{n=0}^{m-1}\left|w_{n}-w_{n+1}\right| \leq K(\mathbf{w}) w_{m}
$$

for all natural numbers $m$, or only for all $m \leq N$ if the sequence w has only finitely many nonzero terms, and the last nonzero term is $w_{N}$.

Another class of sequences, important in applications, has been introduced in [9]. The sequence $\mathbf{w}$ of non-negative numbers tending to zero is called a Mean Rest Bounded Variation Sequence, or briefly $\mathbf{w} \in M R B V S$, if

$$
\sum_{k=m}^{\infty}\left|w_{k}-w_{k+1}\right| \leq \frac{K(\mathbf{w})}{m+1} \sum_{k \in[m / 2, m]} w_{k}
$$

for all natural numbers $m$, where $K(\mathbf{w})$ is a positive constant depending only on w.

The sequence $\mathbf{w}$ of non-negative numbers is called a Mean Head Bounded Variation Sequence (see [16]), or briefly $\mathbf{w} \in M H B V S$, if it has the property

$$
\sum_{k=0}^{n-m-1}\left|w_{k}-w_{k+1}\right| \leq \frac{K(\mathbf{w})}{m+1} \sum_{k=n-m}^{n} w_{k}
$$

for all positive integers $m<n$, where the sequence $\mathbf{w}$ has only finitely many nonzero terms and the last nonzero term is $w_{n}$, and $K(\mathbf{w})$ is a positive constant which depends only on the sequence $\mathbf{w}$.

We see that in the above inequalities a positive constant $K(\mathbf{w})$ is appearing and, for the reason that it has to be finite, we need to suppose the condition $0<K(\mathbf{w}) \leq K$, where $K>0$ is a real number.

It was pointed out in [16] (see also [10]) that

$$
R B V S \subsetneq M R B V S \text { and } H B V S \subsetneq M H B V S .
$$

These sub-classes of the class $\mathbf{S}$ have been used widely in the studies of error estimates through trigonometric Fourier approximation and they still receive considerable attention. Further, we are not going to list all results obtained by others, however for our purposes we are going to write down only those that are connected to ours presented in this research paper.

Let $f(x)$ be a $2 \pi$-periodic continuous function. Let $s_{n}(f):=s_{n}(f ; x)$ denote the $n$-th partial sum of its Fourier series

$$
\frac{a_{0}}{2}+\sum_{k=1}^{\infty}\left(a_{k} \cos k x+b_{k} \sin k x\right)
$$


at $x$, i.e.,

$$
s_{n}(f ; x)=\frac{a_{0}}{2}+\sum_{k=1}^{n}\left(a_{k} \cos k x+b_{k} \sin k x\right),
$$

and let $\omega(\delta)=\omega(\delta, f)$ denote the modulus of continuity of $f$.

Let $A:=\left(a_{n, k}\right)(k, n=0,1, \ldots)$ be a lower triangular infinite matrix of real numbers and let the $A$-transform of $\left\{s_{n}(f ; x)\right\}$ be given by

$$
T_{n, A}(f):=T_{n, A}(f ; x):=\sum_{k=0}^{n} a_{n, k} s_{k}(f ; x) \quad(n=0,1, \ldots) .
$$

The deviation

$$
\left\|T_{n, A}(f)-f\right\|=\sup _{0 \leq x \leq 2 \pi}\left|T_{n, A}(f ; x)-f(x)\right|
$$

was estimated by Chandra $[3,4]$ for monotonic sequences $\left\{a_{n, k}\right\}_{k=0}^{n}$.

Theorem 1. Let $\left\{a_{n, k}\right\}$ satisfy the conditions

$$
\begin{gathered}
a_{n, k} \geq 0 \quad \text { and } \quad \sum_{k=0}^{n} a_{n, k}=1, \\
a_{n, k} \leq a_{n, k+1} \quad(k=0,1, \ldots, n-1 ; n=1,2, \ldots) .
\end{gathered}
$$

Suppose $\omega(t)$ is such that

$$
\int_{u}^{\pi} t^{-2} \omega(t) d t=O(H(u)) \quad(u \rightarrow+0),
$$

where $H(u) \geq 0$ and

$$
\int_{0}^{t} H(u) d u=O(t H(t)) \quad(t \rightarrow+0) .
$$

Then

$$
\left\|T_{n, A}(f)-f\right\|=O\left(a_{n, n} H\left(a_{n, n}\right)\right) .
$$

Theorem 2. Let (2), (3), and (4) hold. Then

$$
\left\|T_{n, A}(f)-f\right\|=O(\omega(\pi / n))+O\left(a_{n, n} H(\pi / n)\right) .
$$

If, in addition, $\omega(t)$ satisfies (5), then

$$
\left\|T_{n, A}(f)-f\right\|=O\left(a_{n, n} H(\pi / n)\right) .
$$

Theorem 3. Let us assume that (2) and

$$
a_{n, k} \geq a_{n, k+1} \quad(k=0,1, \ldots, n-1 ; n=1,2, \ldots)
$$

hold. Then

$$
\left.\left\|T_{n, A}(f)-f\right\|=O\left(\omega(\pi / n)+\sum_{k=1}^{n} k^{-1} \omega(\pi / k) \sum_{r=0}^{k+1} a_{n, r}\right)\right) .
$$


Theorem 4. Let (2), (4), (5), and (6) hold. Then

$$
\left\|T_{n, A}(f)-f\right\|=O\left(a_{n, 0} H\left(a_{n, 0}\right)\right) .
$$

The same results are obtained in [8], when the conditions (3) and (6) on the entries $a_{n, k}$ of the matrix $A$, used in Theorem 1 and Theorem 3 , are replaced, respectively, by conditions

$$
\sum_{k=m}^{\infty}\left|a_{n, k}-a_{n, k+1}\right| \leq K a_{n, m}
$$

and

$$
\sum_{k=0}^{m-1}\left|a_{n, k}-a_{n, k+1}\right| \leq K a_{n, m}
$$

for all natural numbers $m$ with $0 \leq m<n\left(\sum_{p}^{q}(\cdot)=0\right.$ if $\left.p>q\right)$, and of course retaining all other assumed conditions. These results are discussed further in [17], where the conditions (7) and (8) are removed, and simultaneously it is shown that the previous results are consequences of them. Later on, Theorems 1-4 are extended for the so-called sequence-to-sequence transformations (see [5]) and are generalized further in [6] using the absolute values of finite differences of higher order of the entries $a_{n, k}, k=0,1, \ldots, n\left(\Delta^{0} a_{n, k}:=a_{n, k}\right.$, $\Delta^{1} a_{n, k}:=a_{n, k}-a_{n, k+1}, \Delta^{s} a_{n, k}:=\Delta\left(\Delta^{s-1} a_{n, k}\right), s=1,2, \ldots$, and we write $\Delta a_{n, k}$ instead of $\left.\Delta^{1} a_{n, k}\right)$.

Let us suppose that $\mathbb{F}$ is a subset of $\mathbb{N}$ and consider $\mathbb{F}$ as the range of a strictly increasing sequence of positive integers, say $\mathbb{F}=\{\lambda(n)\}_{1}^{\infty}$. Throughout this paper we use the transformation (see [12], [13])

$$
\tau_{n, A}^{\lambda}(f):=\tau_{n, A}^{\lambda}(f ; x):=\sum_{k=0}^{\lambda(n)} a_{\lambda(n), k} s_{k}(f ; x) \quad(n=0,1, \ldots),
$$

where $\lambda(0)=0$,

$$
\begin{gathered}
a_{\lambda(n), k} \geq 0, \quad k=0,1, \ldots, \lambda(n), \\
\sum_{k=0}^{\lambda(n)} a_{\lambda(n), k}=1 .
\end{gathered}
$$

Also, we write

$$
\begin{gathered}
s_{k}(f ; x)=\frac{1}{2 \pi} \int_{-\pi}^{\pi} f(x+t) \frac{\sin \left(k+\frac{1}{2}\right) t}{2 \sin \frac{t}{2}} d t, \\
A_{\lambda(n), \ell}:=\sum_{k=0}^{\ell} a_{\lambda(n), k},
\end{gathered}
$$

and everywhere $[y]$ denotes the largest integer less than or equal to $y$. 
Now we introduce conditions on $a_{\lambda(n), k}$ which will be used in the sequel. Assume that, for all $\lambda(n)$ and $0 \leq m<\lambda(n)$, conditions

$$
\sum_{k=m}^{\infty}\left|a_{\lambda(n), k}-a_{\lambda(n), k+1}\right| \leq K(m+1)^{-1} \sum_{k=\frac{m}{2}}^{m} a_{\lambda(n), k}
$$

and

$$
\sum_{k=0}^{\lambda(n)-m-1}\left|a_{\lambda(n), k}-a_{\lambda(n), k+1}\right| \leq K(m+1)^{-1} \sum_{k=\lambda(n)-m}^{\lambda(n)} a_{\lambda(n), k}
$$

hold true, where $K$ has the same meaning as previously.

The aim of this paper is to estimate the deviation $\left\|\tau_{n, A}^{\lambda}(f)-f\right\|$ when the entries $\left\{a_{\lambda(n), k}\right\}_{k=0}^{n}$ of $A$, satisfy the conditions (14) and (15) instead of conditions (7) and (8), respectively. As we will see later, in special cases, our results not only contain those proved previously by others, but in some cases, they give a better degree of approximation in the sup-norm.

We write $u=\mathcal{O}(v)$ if there exists a positive constant $C$, such that $u \leq$ $C v$, and we assume that all transformations under consideration are regular transformations even if this is not written out explicitly.

\section{Helpful lemmas}

The next two lemmas have been proved implicitly earlier.

Lemma 1. Let $\{\lambda(n)\}_{n=1}^{\infty}$ be a strictly increasing sequence of positive integers. If (4) and (5) hold, then

$$
\int_{0}^{\frac{\pi}{\lambda(n)}} \omega(t) d t=\mathcal{O}\left(\lambda^{-2}(n) H\left(\frac{\pi}{\lambda(n)}\right)\right) .
$$

Proof. Integrating by parts and using (4) and (5), we obtain

$$
\begin{aligned}
\int_{0}^{\frac{\pi}{\lambda(n)}} \omega(t) d t & =\left.\left(-t^{2} \int_{t}^{\pi} \frac{\omega(z)}{z^{2}} d z\right)\right|_{0} ^{\frac{\pi}{\lambda(n)}}+2 \int_{0}^{\frac{\pi}{\lambda(n)}} t\left(\int_{t}^{\pi} \frac{\omega(z)}{z^{2}} d z\right) d t \\
& =\mathcal{O}\left(\lambda^{-2}(n) H\left(\frac{\pi}{\lambda(n)}\right)+\int_{0}^{\frac{\pi}{\lambda(n)}} t H(t) d t\right) \\
& =\mathcal{O}\left(\lambda^{-2}(n) H\left(\frac{\pi}{\lambda(n)}\right)\right)
\end{aligned}
$$

which proves the lemma.

Remark 1. For $\lambda(n)=n$, Lemma 1 reduces to Lemma 1 of [8]. 
Lemma 2 (see [4]). If (4) and (5) hold, then

$$
\int_{0}^{r} t^{-1} \omega(t) d t=\mathcal{O}(r H(r)) \quad(r \rightarrow+0)
$$

Lemma 3. If, for a fixed $\lambda(n)$, the sequence $\left\{a_{\lambda(n), k}\right\}_{k=0}^{n}$ satisfies condition (14), then uniformly in $0<t \leq \pi$,

$$
\left|\mathbb{B}_{\lambda(n), 0}(t)\right|:=\left|\sum_{k=0}^{\lambda(n)} a_{\lambda(n), k} \sin \left(k+\frac{1}{2}\right) t\right|=\mathcal{O}\left(\ell A_{\lambda(n), \ell}\right)
$$

where $\ell=[\pi / t]$.

If $\left\{a_{\lambda(n), k}\right\}_{k=0}^{n}$ satisfies condition (15), then

$$
\left|\mathbb{B}_{\lambda(n), 0}(t)\right|=\mathcal{O}\left(\frac{a_{\lambda(n), \lambda(n)}}{t}\right)
$$

Proof. Let us denote

$$
\mathbb{B}_{\lambda(n), m}(t):=\sum_{k=m}^{\lambda(n)} a_{\lambda(n), k} \sin \left(k+\frac{1}{2}\right) t
$$

The above quantity (using summation by parts) can be transformed as

$$
\begin{aligned}
\mathbb{B}_{\lambda(n), m}(t) & \frac{1}{2}\left[-\sum_{k=m}^{\lambda(n)-1} \Delta a_{\lambda(n), k} \sum_{j=1}^{k} \sin \left(j+\frac{1}{2}\right) t\right. \\
& \left.-a_{\lambda(n), m} \sum_{j=1}^{m-1} \sin \left(j+\frac{1}{2}\right) t+a_{\lambda(n), \lambda(n)} \sum_{j=1}^{\lambda(n)} \sin \left(j+\frac{1}{2}\right) t\right] \\
= & \frac{1}{2 \sin \frac{t}{2}}\left[-\sum_{k=m}^{\lambda(n)-1} \Delta a_{\lambda(n), k}(\cos t-\cos (k+1) t)\right. \\
& \left.-a_{\lambda(n), m}(\cos t-\cos (m t))+a_{\lambda(n), \lambda(n)}(\cos t-\cos (\lambda(n)+1) t)\right] .
\end{aligned}
$$


By $a_{\lambda(n), m} \geq 0$, supposing that $\lambda(n) \geq \ell$, we have

$$
\begin{aligned}
& \left|\mathbb{B}_{\lambda(n), 0}(t)\right| \\
& \quad \leq \sum_{k=0}^{\ell} a_{\lambda(n), k}+\left|\mathbb{B}_{\lambda(n), \ell}(t)\right| \\
& \leq A_{\lambda(n), \ell}+\frac{\pi}{t}\left[\sum_{k=\ell}^{\lambda(n)-1}\left|\Delta a_{\lambda(n), k}\right|+a_{\lambda(n), \ell}+a_{\lambda(n), \lambda(n)}\right] \\
& \quad \leq A_{\lambda(n), \ell}+(\ell+1)\left[K(\ell+1)^{-1} \sum_{k \in[\ell / 2, \ell]} a_{\lambda(n), k}+a_{\lambda(n), \ell}+a_{\lambda(n), \lambda(n)}\right] \\
& \quad \leq(K+1) A_{\lambda(n), \ell}+(\ell+1)\left[a_{\lambda(n), \ell}+a_{\lambda(n), \lambda(n)}\right] .
\end{aligned}
$$

Moreover, it is obvious that

$$
a_{\lambda(n), \ell} \leq A_{\lambda(n), \ell}
$$

and since $\left\{a_{\lambda(n), k}\right\}_{k=0}^{n}$ satisfies (14), we get

$$
\begin{aligned}
a_{\lambda(n), \lambda(n)}-a_{\lambda(n), \ell} & \leq \sum_{k=\ell}^{\lambda(n)-1}\left|\Delta a_{\lambda(n), k}\right| \leq \sum_{k=\ell}^{\infty}\left|\Delta a_{\lambda(n), k}\right| \\
& \leq K(\ell+1)^{-1} \sum_{k \in[\ell / 2, \ell]} a_{\lambda(n), k} \leq K(\ell+1)^{-1} A_{\lambda(n), \ell} .
\end{aligned}
$$

Using (19), (20), and (21), we obtain

$$
\left|\mathbb{B}_{\lambda(n), 0}(t)\right| \leq(2 K+1+2(\ell+1)) A_{\lambda(n), \ell}=\mathcal{O}\left(\ell A_{\lambda(n), \ell}\right),
$$

which proves (16).

For $m=0$, since $\sin \beta \geq \frac{2 \beta}{\pi}(\beta \in(0, \pi / 2])$ and $\left\{a_{\lambda(n), k}\right\}_{k=0}^{n}$ satisfies (15), we have

$$
\begin{aligned}
\left|\mathbb{B}_{\lambda(n), m}(t)\right| & =\left|\mathbb{B}_{\lambda(n), 0}(t)\right| \\
& \leq \frac{1}{2 t}\left[a_{\lambda(n), 0}+\sum_{k=0}^{\lambda(n)-1}\left|\Delta a_{\lambda(n), k}\right|+a_{\lambda(n), \lambda(n)}\right] \\
& \leq \frac{1}{2 t}\left[a_{\lambda(n), 0}+K \sum_{k=\lambda(n)}^{\lambda(n)} a_{\lambda(n), k}+a_{\lambda(n), \lambda(n)}\right] \\
& \leq \frac{1}{2 t}\left[a_{\lambda(n), 0}+(K+1) a_{\lambda(n), \lambda(n)}\right] .
\end{aligned}
$$


Note that condition (15) guarantees also the inequality

$$
\begin{aligned}
a_{\lambda(n), 0}-a_{\lambda(n), \lambda(n)} & \leq \sum_{k=0}^{\lambda(n)-1}\left|\Delta a_{\lambda(n), k}\right| \\
& \leq K \sum_{k=\lambda(n)}^{\lambda(n)} a_{\lambda(n), k} \leq K a_{\lambda(n), \lambda(n)}
\end{aligned}
$$

or

$$
a_{\lambda(n), 0} \leq(K+1) a_{\lambda(n), \lambda(n)},
$$

which along with (22) imply

$$
\left|\mathbb{B}_{\lambda(n), 0}(t)\right| \leq \frac{K+1}{t} a_{\lambda(n), \lambda(n)} .
$$

This proves (17)

Remark 2. Because of (1) this lemma holds true even if conditions (14) and (15) are replaced by conditions (7) and (8), respectively.

Remark 3. Note that Lemma 3 proved in [8], is a particular case of Lemma 3 . It is enough to take $\lambda(n)=n$ in it.

Remark 4. To prove our results we use the same technique as did the authors of [3], [4], and [8].

\section{Main results}

Theorem 5. Let $a_{\lambda(n), k},(k=0,1, \ldots, \lambda(n))$ satisfy the conditions (10), (11), and (15). Suppose $\omega(t)$ is such that (4) holds and $H(t)$ satisfies (5). Then

$$
\left\|\tau_{n, A}^{\lambda}(f)-f\right\|=\mathcal{O}\left(a_{\lambda(n), \lambda(n)} H\left(a_{\lambda(n), \lambda(n)}\right)\right) .
$$

Proof. Setting

$$
\phi_{x}(t):=\frac{f(x+t)+f(x-t)-2 f(x)}{2}
$$

and using (9), (11), (12), we can easily verify that the equality

$$
\tau_{n, A}^{\lambda}(f ; x)-f(x)=\frac{2}{\pi} \int_{0}^{\pi} \frac{\phi_{x}(t)}{2 \sin \frac{t}{2}} \mathbb{B}_{\lambda(n), 0}(t) d t
$$

holds true. 
From (23) we have

$$
\begin{aligned}
\left\|\tau_{n, A}^{\lambda}(f)-f\right\| & \leq \frac{2}{\pi} \int_{0}^{\pi} \frac{\omega(t)}{2 \sin \frac{t}{2}}\left|\mathbb{B}_{\lambda(n), 0}(t)\right| d t \\
& \leq \frac{2}{\pi}\left(\int_{0}^{a_{\lambda(n), \lambda(n)}}+\int_{a_{\lambda(n), \lambda(n)}}^{\pi}\right)=: \mathbb{I}_{1}(\lambda(n))+\mathbb{I}_{2}(\lambda(n)) .
\end{aligned}
$$

Based on the inequality

$$
\left|\mathbb{B}_{\lambda(n), 0}(t)\right|=\left|\sum_{k=0}^{\lambda(n)} a_{\lambda(n), k} \sin \left(k+\frac{1}{2}\right) t\right| \leq 1
$$

and Lemma 2, we have

$$
\mathbb{I}_{1}(\lambda(n))=\mathcal{O}(1) \int_{0}^{a_{\lambda(n), \lambda(n)}} \frac{\omega(t)}{t} d t=\mathcal{O}\left(a_{\lambda(n), \lambda(n)} H\left(a_{\lambda(n), \lambda(n)}\right)\right) .
$$

On the other hand, by (5), (17) of Lemma 3, and (4) we obtain

$$
\begin{aligned}
\mathbb{I}_{2}(\lambda(n)) & =\mathcal{O}\left(a_{\lambda(n), \lambda(n)}\right) \int_{a_{\lambda(n), \lambda(n)}}^{\pi} t^{-2} \omega(t) d t \\
& =\mathcal{O}\left(a_{\lambda(n), \lambda(n)} H\left(a_{\lambda(n), \lambda(n)}\right)\right) .
\end{aligned}
$$

Subsequently, by (24), (25), and (26) we arrive at (23) as required.

Theorem 6. Let (10), (11), and (15) be satisfied. If $\omega(t)$ satisfies (4), then

$$
\left\|\tau_{n, A}^{\lambda}(f)-f\right\|=\mathcal{O}\left(\omega\left(\frac{\pi}{\lambda(n)}\right)\right)+\mathcal{O}\left(a_{\lambda(n), \lambda(n)} H\left(\frac{\pi}{\lambda(n)}\right)\right) .
$$

If, in addition, $H(t)$ satisfies (5) then

$$
\left\|\tau_{n, A}^{\lambda}(f)-f\right\|=\mathcal{O}\left(a_{\lambda(n), \lambda(n)} H\left(\frac{\pi}{\lambda(n)}\right)\right) .
$$

Proof. First, we write (24) in the form

$$
\left\|\tau_{n, A}^{\lambda}(f)-f\right\| \leq \frac{2}{\pi}\left(\int_{0}^{\frac{\pi}{\lambda(n)}}+\int_{\frac{\pi}{\lambda(n)}}^{\pi}\right)=: \mathbb{J}_{1}(\lambda(n))+\mathbb{J}_{2}(\lambda(n)) .
$$

Making use of the inequality $|\sin \beta| \leq|\beta|$ and (11), we have

$$
\begin{aligned}
\mathbb{J}_{1}(\lambda(n)) & =\mathcal{O}(1) \int_{0}^{\frac{\pi}{\lambda(n)}} \frac{\omega(t)}{t} \sum_{k=0}^{\lambda(n)} a_{\lambda(n), k}\left(k+\frac{1}{2}\right) t d t \\
& =\mathcal{O}(\lambda(n)) \int_{0}^{\frac{\pi}{\lambda(n)}} \omega(t) d t=\mathcal{O}\left(\omega\left(\frac{\pi}{\lambda(n)}\right)\right) .
\end{aligned}
$$


In order to estimate $\mathbb{J}_{2}(\lambda(n))$, we use again (5), (17) of Lemma 3 , and (4). Indeed, we find that

$$
\begin{aligned}
\mathbb{J}_{2}(\lambda(n)) & =\mathcal{O}\left(a_{\lambda(n), \lambda(n)}\right) \int_{\frac{\pi}{\lambda(n)}}^{\pi} t^{-2} \omega(t) d t \\
& =\mathcal{O}\left(a_{\lambda(n), \lambda(n)} H\left(\frac{\pi}{\lambda(n)}\right)\right) .
\end{aligned}
$$

Thus, (29), (30), and (31) imply (27).

To prove (28) we proceed as follows. Since $\left\{a_{\lambda(n), k}\right\}_{k=0}^{n}$ satisfies condition (15), for $0 \leq v \leq \lambda(n)$ we have

$$
\begin{aligned}
a_{\lambda(n), v}-a_{\lambda(n), \lambda(n)} & \leq \sum_{k=v}^{\lambda(n)-1}\left|\Delta a_{\lambda(n), k}\right| \leq \sum_{k=0}^{\lambda(n)-1}\left|\Delta a_{\lambda(n), k}\right| \\
& \leq K \sum_{k=\lambda(n)}^{\lambda(n)} a_{\lambda(n), k}=K a_{\lambda(n), \lambda(n)} .
\end{aligned}
$$

This implies

whence

$$
a_{\lambda(n), v} \leq(K+1) a_{\lambda(n), \lambda(n)},
$$

$$
(K+1)(\lambda(n)+1) a_{\lambda(n), \lambda(n)} \geq \sum_{v=0}^{\lambda(n)} a_{\lambda(n), v}=1 .
$$

So, using

$$
\frac{1}{\lambda(n)}=\mathcal{O}\left(a_{\lambda(n), \lambda(n)}\right)
$$

and Lemma 1, we get

$$
\begin{aligned}
\mathbb{J}_{1}(\lambda(n)) & =\mathcal{O}(\lambda(n)) \int_{0}^{\frac{\pi}{\lambda(n)}} \omega(t) d t \\
& =\mathcal{O}\left(\frac{1}{\lambda(n)}\right) H\left(\frac{\pi}{\lambda(n)}\right)=\mathcal{O}\left(a_{\lambda(n), \lambda(n)} H\left(\frac{\pi}{\lambda(n)}\right)\right) .
\end{aligned}
$$

Hence, gathering this and (31), we clearly have (28).

Theorem 7. Assume that (10) and (11) hold. If $\left\{a_{\lambda(n), k}\right\}_{k=0}^{n}$ satisfies condition (14), then

$$
\left.\left\|\tau_{n, A}^{\lambda}(f)-f\right\|=\mathcal{O}\left(\omega\left(\frac{\pi}{\lambda(n)}\right)+\sum_{k=1}^{\lambda(n)-1}\left(1+k^{-1}\right) \omega\left(\frac{\pi}{k}\right) \sum_{r=0}^{k+1} a_{\lambda(n), r}\right)\right) .
$$

Proof. We already have proved in Theorem 6 (relation (30)) that

$$
\mathbb{J}_{1}(\lambda(n))=\mathcal{O}\left(\omega\left(\frac{\pi}{\lambda(n)}\right)\right) .
$$


Using (16) of Lemma 3, we have

$$
\begin{aligned}
\mathbb{J}_{2}(\lambda(n)) & =\frac{2}{\pi} \int_{\frac{\pi}{\lambda(n)}}^{\pi} \frac{\omega(t)}{2 \sin \frac{t}{2}}\left|\mathbb{B}_{\lambda(n), 0}(t)\right| d t \\
& =\mathcal{O}(1) \int_{\frac{\pi}{\lambda(n)}}^{\pi} t^{-1} \omega(t) \ell A_{\lambda(n), \ell} d t \\
& =\mathcal{O}(1) \sum_{k=1}^{\lambda(n)-1} \int_{\frac{\pi}{k+1}}^{\frac{\pi}{k}} t^{-1} \omega(t) \ell A_{\lambda(n), \ell} d t \\
& =\mathcal{O}(1) \sum_{k=1}^{\lambda(n)-1} \frac{k+1}{k} \omega(\pi / k) A_{\lambda(n), k+1} .
\end{aligned}
$$

Now from (33), (34), and (29) we obtain (32).

Theorem 8. Let (4), (5), (10), (11), and (14) hold. Then

$$
\left\|\tau_{n, A}^{\lambda}(f)-f\right\|=\mathcal{O}\left(a_{\lambda(n), 0} H\left(a_{\lambda(n), 0}\right)\right) .
$$

Proof. Following (18), we have

$$
\left|\mathbb{B}_{\lambda(n), 0}(t)\right|=\mathcal{O}\left(\frac{1}{t}\right)\left[\sum_{k=0}^{\infty}\left|\Delta a_{\lambda(n), k}\right|+a_{\lambda(n), 0}+a_{\lambda(n), \lambda(n)}\right],
$$

and since, by (14),

$$
a_{\lambda(n), \lambda(n)} \leq \sum_{k=\lambda(n)}^{\infty}\left|\Delta a_{\lambda(n), k}\right| \leq \sum_{k=0}^{\infty}\left|\Delta a_{\lambda(n), k}\right| \leq K a_{\lambda(n), 0},
$$

we find that

$$
\left|\mathbb{B}_{\lambda(n), 0}(t)\right|=\mathcal{O}\left(\frac{a_{\lambda(n), 0}}{t}\right) .
$$

First, we write relation (23) as

$$
\left\|\tau_{n, A}^{\lambda}(f)-f\right\| \leq \frac{2}{\pi}\left(\int_{0}^{a_{\lambda(n), 0}}+\int_{a_{\lambda(n), 0}}^{\pi}\right)=: \mathbb{I}_{1}^{0}(\lambda(n))+\mathbb{I}_{2}^{0}(\lambda(n)) .
$$

Therefore, as in (25), we have

$$
\mathbb{I}_{1}^{0}(\lambda(n))=\mathcal{O}\left(a_{\lambda(n), 0} H\left(a_{\lambda(n), 0}\right)\right) .
$$

Also, by Lemma 3 and (35) we obtain

$$
\begin{aligned}
\mathbb{I}_{2}^{0}(\lambda(n)) & =\mathcal{O}\left(a_{\lambda(n), 0}\right) \int_{a_{\lambda(n), 0}}^{\pi} t^{-2} \omega(t) d t \\
& =\mathcal{O}\left(a_{\lambda(n), 0} H\left(a_{\lambda(n), 0}\right)\right) .
\end{aligned}
$$

Whence, by (36), (37), and (38) we have proved the statement of the theorem. 


\section{Corollaries}

Suppose that $A:=\left(a_{\lambda(n), k}\right)$ is a matrix defined by

$$
a_{\lambda(n), k}= \begin{cases}\frac{p_{\lambda(n)-k}}{P_{\lambda(n)}}, & 0 \leq k \leq \lambda(n), \\ 0 & \text { otherwise }\end{cases}
$$

where $\left\{p_{k}\right\}, k=0,1, \ldots$, is a sequence of non-negative numbers with $P_{\lambda(n)}:=$ $\sum_{k=0}^{\lambda(n)} p_{k} \neq 0$. In this case, the transform $\tau_{n, A}^{\lambda}(f ; x)$ reduces to a polynomial (see [11], page 2) of the form

$$
\tau_{n, A}^{\lambda}(f ; x) \equiv N_{n}^{\lambda}(f ; x):=\frac{1}{P_{\lambda(n)}} \sum_{k=0}^{\lambda(n)} p_{\lambda(n)-k} s_{k}(f ; x) .
$$

Whence, Theorem 5 implies the following corollary.

Corollary 1. Let (4) and (5) hold. If $\left\{p_{k}\right\}$ satisfies condition (15), then

$$
\left\|N_{n}^{\lambda}(f)-f\right\|=\mathcal{O}\left(\frac{p_{\lambda(n)}}{P_{\lambda(n)}} H\left(\frac{p_{\lambda(n)}}{P_{\lambda(n)}}\right)\right) .
$$

Consider the matrix $A:=\left(a_{\lambda(n), k}\right)$ with

$$
a_{\lambda(n), k}= \begin{cases}\frac{p_{k}}{P_{\lambda(n)}}, & 0 \leq k \leq \lambda(n), \\ 0 & \text { otherwise, }\end{cases}
$$

where $\left\{p_{k}\right\}, k=0,1, \ldots$, is a sequence of non-negative numbers with $P_{\lambda(n)}:=\sum_{k=0}^{\lambda(n)} p_{k} \neq 0$. Now, the transform $\tau_{n, A}^{\lambda}(f ; x)$ is the polynomial (see [11], page 3)

$$
\tau_{n, A}^{\lambda}(f ; x) \equiv R_{n}^{\lambda}(f ; x):=\frac{1}{P_{\lambda(n)}} \sum_{k=0}^{\lambda(n)} p_{k} s_{k}(f ; x) .
$$

So, in this case we have the following result.

Corollary 2. Let (4) and (5) hold. If $\left\{p_{k}\right\}$ satisfies condition (15), then

$$
\left\|R_{n}^{\lambda}(f)-f\right\|=\mathcal{O}\left(\frac{p_{\lambda(n)}}{P_{\lambda(n)}} H\left(\frac{p_{\lambda(n)}}{P_{\lambda(n)}}\right)\right) .
$$

In particular, if $\omega(u)=u^{\alpha}, 0<\alpha \leq 1$, and $f \in \operatorname{Lip}(\omega) \equiv \operatorname{Lip}(\alpha)$, then $\omega(t)=\mathcal{O}\left(\delta^{\alpha}\right), \delta \geq 0$. Thus, if $f \in \operatorname{Lip}(\alpha)$ and

$$
H(u)= \begin{cases}u^{\alpha-1}, & 0<\alpha<1 \\ \log \left(\frac{\pi}{u}\right), & \alpha=1\end{cases}
$$

then from Corollary 2 we get a version of a theorem proved in [2]. 
Corollary 3. Let $f \in C[0,2 \pi], f \in \operatorname{Lip}(\alpha),(0<\alpha \leq 1)$, and $\left\{p_{k}\right\} \in$ $M H B V S$. Then the degree of approximation of $f$ by $R_{n}^{\lambda}(f ; x)$ of its Fourier series is given by

$$
\left\|R_{n}^{\lambda}(f)-f\right\|= \begin{cases}\mathcal{O}\left(\left(\frac{p_{\lambda(n)}}{P_{\lambda(n)}}\right)^{\alpha}\right), & 0<\alpha<1, \\ \mathcal{O}\left(\frac{p_{\lambda(n)}}{P_{\lambda(n)}} \log \left(\frac{\pi P_{\lambda(n)}}{p_{\lambda(n)}}\right)\right), & \alpha=1 .\end{cases}
$$

Remark 5. Note that for $\lambda(n)=n$ the means $R_{n}^{\lambda}(f ; x)$ reduce to wellknown Riesz means $R_{n}(f ; x)$. In the case when $\left\{p_{k}\right\}$ is a positive and nondecreasing sequence, Corollary 3 has been proved earlier in [2].

If the matrix $A:=\left(a_{\lambda(n), k}\right)$ is defined by (see [1], page 195))

$$
a_{\lambda(n), k}= \begin{cases}\frac{1}{\lambda(n)+1}, & 0 \leq k \leq \lambda(n) \\ 0 & \text { otherwise }\end{cases}
$$

then

$$
\tau_{n, A}^{\lambda}(f ; x) \equiv C_{n}^{\lambda}(f ; x):=\frac{1}{\lambda(n)+1} \sum_{k=0}^{\lambda(n)} s_{k}(f ; x) .
$$

Consequently, Corollary 2 implies the following.

Corollary 4. Let $f \in C[0,2 \pi]$ and $f \in \operatorname{Lip}(\alpha),(0<\alpha \leq 1)$. Then the degree of approximation of $f$ by $C_{n}^{\lambda}(f ; x)$ of its Fourier series is given by

$$
\left\|C_{n}^{\lambda}(f)-f\right\|= \begin{cases}\mathcal{O}\left((\lambda(n)+1)^{-\alpha}\right), & 0<\alpha<1, \\ \mathcal{O}\left(\frac{\log (\pi(\lambda(n)+1))}{\lambda(n)+1}\right), & \alpha=1 .\end{cases}
$$

Remark 6. Since $\lambda(n) \geq n$ for all $n$, the means $C_{n}^{\lambda}(f ; x)$ in Corollary 4 , give a degree of approximation not worse than classical Cesàro means $C_{n}(f ; x)$.

Remark 7. Note that, putting $\lambda(n)=n$ in Corollary 4, we obtain a wellknown result given in [15], page 13 .

Remark 8. Analogous result, with one given in [14], can be derived from Theorem 7 .

Remark 9. Putting $\lambda(n)=n$ for all $n$, and specifying particular conditions on the matrix $A$, all theorems proved in [3] and [8] are consequences of ours.

\section{Conclusions}

Approximation of periodic and continuous functions by trigonometric polynomials has taken a great place in approximation theory. Approximation of such functions has been done in different norms and particularly in sup-norm, using various transforms of their partial sums of their Fourier 
series. Such transforms are realized by a particular matrix whose entries belong to various classes of real sequences. In this research paper, we have used regular transformations of the form

$$
\tau_{n, A}^{\lambda}(f):=\tau_{n, A}^{\lambda}(f ; x):=\sum_{k=0}^{\lambda(n)} a_{\lambda(n), k} s_{k}(f ; x), \quad(n=0,1, \ldots),
$$

by a matrix whose entries belong to the Mean Rest Bounded Variation Sequences or the Mean Head Bounded Variation Sequences, proving some new results pertaining to the degree of approximation in the sup-norm of periodic continuous functions. All results are expressed in terms of an auxiliary function $H(t) \geq 0$ and in terms of entries of a matrix whose indices form a strictly increasing sequence of positive integers $\lambda:=\{\lambda(n)\}_{n=1}^{\infty}$, which in general, give a degree of approximation not worse than some means used previously by other authors.

\section{Acknowledgements}

The author would like to thank the anonymous referee for her/his valuable remarks and comments, which definitely contributed to the quality of this paper.

\section{References}

[1] D. H. Armitage and I. J. Maddox, A new type of Cesàro mean, Analysis 9(1-2) (1989), 195-204.

[2] P. Chandra, On the degree of approximation of functions belonging to the Lipschitz class, Nanta Math. 8 (1975), 88-91.

[3] P. Chandra, On the degree of approximation of a class of functions by means of Fourier series, Acta Math. Hungar. 52 (1988), 199-205.

[4] P. Chandra, A note on the degree of approximation of continuous functions, Acta Math. Hungar. 62 (1993), 21-23.

[5] Xh. Z. Krasniqi, On the degree of approximation of continuous functions that pertains to the sequence-to-sequence transformation, Aust. J. Math. Anal. Appl. 7(2) (2010), Art. $13,10 \mathrm{pp}$

[6] Xh. Z. Krasniqi, On the degree of approximation of continuous functions by matrix means related to partial sums of a Fourier series, Comment. Math. 52(2) (2012), 207215.

[7] L. Leindler, On the uniform convergence and boundedness of a certain class of sine series, Anal. Math. 27(4) (2001), 279-285.

[8] L. Leindler, On the degree of approximation of continuous functions, Acta Math. Hungar. 104 (2004), 105-113.

[9] L. Leindler, Integrability conditions pertaining to Orlicz space, JIPAM. J. Inequal. Pure Appl. Math. 8(2) (2007), Article 38, 6 pp.

[10] W. Lenski, and B. Szal, Pointwise approximation of functions from $L^{p}(w)_{\beta}$ by linear operators of their Fourier series, J. Funct. Spaces Appl. 2012, Art. ID 930967, 16 pp.

[11] M. L. Mittal and M. V. Singh, Approximation of signals (functions) by trigonometric polynomials in $L_{p}$-norm, Int. J. Math. Math. Sci. 2014, Art. ID 267383, 6 pp. 
[12] M. L. Mittal and M. V. Singh, Approximation of functions of slass Lip $(\alpha, p)$ in $L_{p^{-}}$ norm, in: Springer Proceedings in Mathematics and Statistics 143, 2015, pp. 109-120.

[13] M. L. Mittal and M. V. Singh, Applications of Cesàro submethod to trigonometric approximation of signals (functions) belonging to class $\operatorname{Lip}(\alpha, p)$ in $L_{p}$-norm, J. Math. 2016, Art. ID 9048671, 7 pp.

[14] R. N. Mohapatra and P. Chandra, Degree of approximation of functions in the Holder metric, Acta Math. Hungar. 41(1-2) (1983), 67-76.

[15] B. N. Sahney and R. V. Venugopal, Error bounds in the approximation of functions, Bull. Austral. Math. Soc. 6 (1972), 11-18.

[16] B. Szal, A note on the uniform convergence and boundedness of a generalized class of sine series, Comment. Math. 48 (2008), no. 1, 85-94.

[17] B. Wei and D. Yu, On the degree of approximation of continuous functions by means of Fourier series, Math. Commun. 17 (2012), 211-219.

University of Prishtina "Hasan Prishtina", Faculty of Education, Avenue "Mother Theresa", 10000 Prishtina, Republic of Kosovo

E-mail address: xhevat.krasniqi@uni-pr.edu 